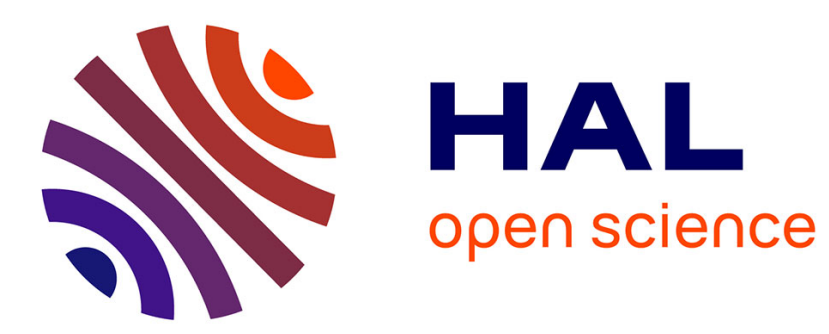

\title{
Transsexualisme/ transgenderism : psychothérapie et transformation hormonale et chirurgicale
}

\author{
Jean-Baptiste Marchand, Elise Pelladeau, François Pommier
}

\section{To cite this version:}

Jean-Baptiste Marchand, Elise Pelladeau, François Pommier. Transsexualisme/ transgenderism : psychothérapie et transformation hormonale et chirurgicale. Annales Médico-Psychologiques, Revue Psychiatrique, 2017, 175 (3), pp.210-216. 10.1016/j.amp.2016.05.016 . hal-03211688

\section{HAL Id: hal-03211688 \\ https://hal.science/hal-03211688}

Submitted on 30 Apr 2021

HAL is a multi-disciplinary open access archive for the deposit and dissemination of scientific research documents, whether they are published or not. The documents may come from teaching and research institutions in France or abroad, or from public or private research centers.
L'archive ouverte pluridisciplinaire HAL, est destinée au dépôt et à la diffusion de documents scientifiques de niveau recherche, publiés ou non, émanant des établissements d'enseignement et de recherche français ou étrangers, des laboratoires publics ou privés. 


\title{
Transsexualisme/transgenderism : Psychothérapie et Transformation hormonale et chirurgicale
}

\author{
Transsexualism/transgenderism: Psychotherapy \\ and Sex reassignment therapy
}

Jean-Baptiste Marchand

Elise Pelladeau

François Pommier

\section{RÉSUMÉ}

A l'issue des évaluations françaises et internationales concernant la prise en charge des patients transsexuels (et transgenres), l'intérêt de proposer une psychothérapie en complément de la transformation hormono-chirurgicale, a été unanimement reconnu. Force est de constater que les travaux concernant ce sujet sont rares, que les modalités de cette proposition restent mal définies, et que sur ce sujet des questions restent en suspens. Cet article propose donc de présenter la conception et l'évolution de cette psychothérapie dans les différentes versions des standards de soins, ainsi que les approches théoriques sur lesquelles le modèle actuel est fondé, puis d'exposer les questions qui persistent selon nous, concernant ce sujet, ainsi que nos remarques et propositions se rapportant à ces dernières.

Mots-clés : $\quad$ Transsexualisme, Dysphorie de genre, Standards de soins, Transgenderism, Psychothérapie, Transformation hormonale et chirurgicale (THC), World Professional Association for Transgender Health (WPATH).

\section{ABSTRACT \\ Title : Transsexualism/transgenderism: Psychotherapy and Sex reassignment therapy}

At the end of the French and international evaluations concerning the care of transsexual (and transgender) patients, the interest to propose a psychotherapy as a supplement to the sex reassignment therapy, was unanimously recognized. It is clear that the works concerning this subject are rare, that the methods of this proposal remain ill-defined, and that some questions remain unsettled on this subject. The goal of this article is to present the conception and the evolution of this psychotherapy in the various versions of the standards of care, as well as the theoretical approaches on which the current model is established, then to expose the questions which according to us, still persist concerning this subject, as well as our remarks and proposals relating to the latter.

Keywords : Transsexualism, Gender Dysphoria, Standards of care (SOC), Transgenderism, Psychotherapy, Sex reassignment therapy (SRT),

World Professional Association for Transgender Health (WPATH).

\section{EXTENDED ABSTRACT}

\section{Objectives}

At the end of the French evaluations and the international revision concerning the standard of care (SOC) for transsexual and transgender patients, the interest to propose psychotherapy as 
a supplement to the sex reassignment therapy (SRT), was unanimously recognized. But, it is clear that the works concerning this subject are rare, and that the methods of this proposal remain ill-defined. So, many questions remain unsettled such its frame, modalities, methods, objectives and its place and relation with or in the SRT, as well as on the theoretical models on which these psychotherapies base themselves. The goal of this article is therefore to present the conception and the evolution of this psychotherapy in the various versions of the standards of care, as well as the theoretical approaches on which the current model is established, then to expose the questions which according to us, still persist concerning this subject, as well as our remarks and proposals relating to the latter.

\section{Materials}

At start, with the first SRT in the 50's, this practice and his combination with psychotherapy suffered from a strong ethical but also partially ideological, controversy. An important number of medical practitioners met around the endocrinologist $\mathrm{H}$. Benjamin to establish the first standards of care by SRT for transsexual, and defended that this treatment was the only one that could relieve the problem of gender identity of the transsexual patients. In the opposite, some psychiatrists and psychologists were in strict opposition to this treatment and postulated that transsexualism was a psychotic disorder which needed to be cured only by psychotherapy. In spite of this fist hard confrontation, it seems accepted today that the SRT can bring beneficial effects, and that an additional psychotherapy is maximizing the prognostic of this treatment. So, in the present, in the SOC, the goal of psychotherapy is "to help transsexual, transgender [...] individuals achieve long-term comfort in their gender identity expression,...". However, the question of the modalities, forms and theoretical fundaments stay still not well defined.

\section{Results}

Today, the SOC recommend that psychotherapist actively collaborate and participate to the decision-making reunions of the SRT's professional team. In the same way, the role of the psychotherapist is described very near of a role of coaching and counseling, to optimize the SRT's final result. In complete consistency with these points, the theoretical models exposed are unconflict and unpathologized models which postulate that the true gender of patients couldn't be expressed because of social stigmatizations which can make them present a gender dysphoria. But, these points of view are more controversial that it's appearing.

\section{Conclusions}

The more controversial point concerns the narrow collaboration and the active participation of the psychotherapist with the SRT's professional team and its potential consequences. In that configuration, a collusion of the different therapeutic spaces can take place. The patients can feel unease with this particular therapeutic relation. In the same way, on the theoretical level, the conflict is a primary fundament in a psychodynamic perspective, but it also can be considered as a normal element of the therapy and the SRT's way. For all of those remarks, the question and works on the psychotherapy in complement with the SRT have to be continued. 


\section{Introduction}

Ces dernières années, le protocole de prise en charge des patients transsexuels par Transformation Hormonale et Chirurgicale (THC) a fait l'objet de plusieurs expertises, évaluations et révisions. En France, à la demande du Ministère de la Santé, la Haute Autorité de Santé (HAS) a publié en 2009 un premier rapport concernant l'état de la prise en charge du transsexualisme en France [17], suivi par un second rapport rédigé par l'Inspection Générale des Affaires Sociales (IGAS) en 2011 [18]. En parallèle, au niveau international, la World Professionnal Association for Transgender Health (WPATH) travaillait sur la septième version de ses standards de soins qu'elle a finalement publiée en 2011, et opérait un changement majeur de ceux-ci. En effet, conformément au changement de nom de cette association en 2007, cette septième version des standards de soins a marqué le passage du paradigme clinique transsexuel à celui transgenre. Cette septième version est fondée non plus sur un modèle binaire de l'identité sexuée (ou de genre) masculine ou féminine, mais sur la conception défendue par Coleman et al. d'un spectre du genre prenant en considération les multiples variations de celui-ci et acceptant les non-conformités de genre [8].

Cependant, dans tous ces travaux sur les protocoles de soins par THC, nous pouvons constater que cinq types de soins majeurs sont mis en avant :

1) l'Etape de diagnostic et d'évaluation,

2) l'Expérience en vie réelle ${ }^{1}$,

3) l'Hormonosubstitution,

4) la Chirurgie de réassignation des organes génitaux

et 5) la Psychothérapie (ou accompagnent psychothérapeutique).

Or, parmi ceux-ci, l'expérience en vie réelle et la psychothérapie n'ont pas le même statut d'étape «incontournable », que l'étape du diagnostic et de l'évaluation qui est considérée comme obligatoire, et que celles de l'hormonosubstitution et de la chirurgie de réassignation qui sont l'objet central de la demande des patients aux médecins et les étapes fondamentales et constituantes de la THC. En effet, selon les standards de soins de la WPATH, le choix d'entreprendre l'expérience en vie réelle ou une psychothérapie doit revenir entièrement au patient, et ne doit en aucun cas lui être imposées. Elles sont fortement conseillées car considérées comme pouvant améliorer le bon déroulement et l'aboutissement de la THC, mais elles ne sont pas des conditions nécessaires à la poursuite de la transformation.

Cependant, à propos de la psychothérapie des personnes transsexuelles et transgenres, force est de constater que ce sujet est délicat, et que peu d'articles lui sont consacrés. Ainsi,

\footnotetext{
${ }^{1}$ Real life test : moment où le patient passe le cap de vivre et d'adopter en permanence le rôle du sexe revendiqué et ce, dans toutes les activités quotidiennes sociales et professionnelles afin que le patient puisse tester sa capacité à vivre dans le rôle du sexe désiré et la persistance de sa demande avant d'entamer les étapes irréversibles de la transformation dont tout particulièrement la chirurgie de réassignation.
} 
Marchand J-B. et al. (2017). Transsexualisme/transgenderism : psychothérapie et transformation hormonale et chirurgicale. Annales Médico-Psychologiques, 175 (3), 210-216. http://dx.doi.org/10.1016/j.amp.2016.05.016

beaucoup de questions restent en suspens tels son cadre, ses modalités, son objectif et sa place et sa relation à la THC, ainsi que sur les modèles théoriques sur lesquels se basent ces psychothérapies.

Pour cette raison, après une présentation résumant l'histoire de la THC et de son rapport à la psychothérapie des patients transsexuels, cet article présentera les recommandations actuelles des standards de soins de la WPATH concernant la psychothérapie des patients transsexuels et transgenres, puis proposera dans un second temps des pistes nous ayant semblé pertinentes pour poursuivre le travail de réflexion sur l'élaboration d'un cadre psychothérapeutique pour la prise en charge de ces patients poursuivant ou non une THC.

\section{La transformation hormonale et chirurgicale : réponse médicale au vœu transsexuel}

\subsection{Premières Transformations Hormonales et Chirurgicales et constitution des Standards de soins}

Les premières tentatives de THC généralement reconnues ont eu lieu à Berlin, durant la première moitié du XIX ${ }^{\text {ème }}$ siècle [6], avec des essais cliniques de greffes chirurgicales d'organes et de glandes endocriniennes l'expérience en vie réelle et la psychothérapie. Cependant, ce n'est que dans les années 50 que le cas très médiatisé de George devenu Christine Jorgensen ${ }^{2}$, en tant que premier cas de THC considérée comme réussie, inaugure la prise en charge des patients transsexuels par THC. Suite à celle-ci, le nombre de demandes de changement de sexe va fortement augmenter, mais la THC va aussi servir à l'endocrinologue $\mathrm{H}$. Benjamin pour donner sa première définition au transsexualisme :

«[Pour les patients transsexuels] leurs organes, primaires (testicules) comme secondaires (pénis et autres), sont de dégoutantes difformités devant être changées par le bistouri du chirurgien... Ce n'est qu'à cause de récentes et grandes avancées de l'endocrinologie et des techniques chirurgicales que le tableau a changé » [2, p. 11].

Encore actuellement, même si l'intérêt de distinguer la demande de changement de sexe des patients, de la réponse du corps médical qu'est la THC, est reconnu, l'OMS et l'APA octroient la même importance à la THC dans la sémiologie du syndrome transsexuel :

"...désir de vivre et d'être accepté en tant que personne appartenant au sexe opposé. Ce désir s'accompagne habituellement [...] du souhait de subir une intervention chirurgicale et un traitement hormonal afin de rendre son corps aussi conforme que possible au sexe désiré. » [29, p. 123]

et «...2- Désir persistant d'être débarrassé des caractéristiques sexuelles primaires et secondaires du sexe biologique. 3- Fort désir de posséder les caractéristiques sexuelles primaires et/ou secondaires de l'autre genre. » [1]

${ }^{2}$ Le New York Daily News du $1^{\text {er }}$ décembre 1952 titre en première page, «Un ex-GI devient une ravissante blonde ». 
Marchand J-B. et al. (2017). Transsexualisme/transgenderism : psychothérapie et transformation hormonale et chirurgicale. Annales Médico-Psychologiques, 175 (3), 210-216. http://dx.doi.org/10.1016/j.amp.2016.05.016

\subsection{Psychothérapie ou Transformation Hormonale et Chirurgicale : de la confrontation à la conjugaison}

Encore aujourd'hui, l'origine de la problématique identitaire des patients transsexuels reste inconnue. En cela, selon les points de vue, la THC peut être considérée comme un soin palliatif pouvant être bénéfique et pouvant soulager ces patients, plutôt qu'en tant que soin curatif. Pour cette raison mais aussi du fait de son caractère spectaculaire (voire extrême pour certains) et qu'elle représente un véritable défi au principe de déontologie médicale de primum non nocere $^{3}$, le recours à la THC ne va pas de soi, et invite à la précaution. Dans cette situation délicate, dans un premier temps, une confrontation a eu lieu mettant en opposition les recours à la THC ou à une psychothérapie, avant que cette mise en opposition ne s'apaise et que seulement dans un second temps, une conjugaison des deux approches thérapeutiques ne soit admise et proposée dans la prise en charge.

Ainsi, suite à la médiatisation de la THC de Christine Jorgensen, une partie importante du corps médical inspirée et menée par Harry Benjamin et ses travaux, va considérer que le transsexualisme est un problème constitutionnel qui a certainement une origine biologique même si celle-ci n'est pas décelable par les moyens de l'époque, et que l'identification du patient au sexe opposé est une empreinte indélébile qui doit être considérée comme normale. Selon ce point de vue, le recours à une psychothérapie de conversion vers une identité sexuée en adéquation avec le sexe organique n'aurait aucun effet, et serait même totalement inutile. Pour ceux-ci, la THC serait donc la seule solution, le seul «traitement», pouvant soulager la problématique transsexuelle de ces patients [2].

En France, les premières équipes pluridisciplinaires pratiquant des THC se sont constituées dans les années 70-80, et jusque dans les années 2000, un nombre important de psychiatres et psychologues psychanalystes se sont montrés fortement opposés à la THC [9-10, 14, 27]. Selon ces derniers, le transsexualisme relèverait de la psychose, et le recours à la THC ne pourrait aboutir qu'au suicide et à la décompensation psychotique de ces patients. Selon ces auteurs, la psychothérapie (psychanalytique ou non) serait la solution à privilégier pour la prise en charge des patients transsexuels, et il s'agirait par celle-ci de traiter directement la problématique identitaire des patients. L'objectif de celle-ci serait donc d'amener ces derniers à réinvestir leur corps et une identité sexuée en adéquation avec celui-ci. Ainsi, en France, dans un premier temps, nous assistons donc à une controverse assez virulente opposant les recours à la THC ou à la psychothérapie.

Cependant, en-dehors de ces deux positions clivées, la combinaison d'un recours à la THC et d'une psychothérapie au sein du même protocole de prise en charge du transsexualisme s'est établie. En effet, actuellement, cette controverse opposant psychothérapie et THC tend à s'apaiser. Les travaux sur le devenir des patients transsexuels concluent que le recours à la THC donne lieu à des effets pouvant être bénéfiques aux patients et que les regrets, insatisfactions et échecs sont plus rares que ce que prédisaient les opposants à la THC [25]. Ainsi, de nos jours, il est admis que selon certaines conditions de diagnostic et d'évaluation, le recours à la THC peut apporter des effets bénéfiques aux patients et, qu'un travail psychothérapeutique en association à celle-ci, peut être bénéfique à ces derniers.

Plus précisément, aux Etats-Unis puis au niveau international, dès leur première édition en 1979, les standards de soins de la WPATH ont comporté une partie recommandant un

\footnotetext{
${ }^{3}$ D'abord ne pas nuire.
} 
Marchand J-B. et al. (2017). Transsexualisme/transgenderism : psychothérapie et transformation hormonale et chirurgicale. Annales Médico-Psychologiques, 175 (3), 210-216. http://dx.doi.org/10.1016/j.amp.2016.05.016

accompagnement psychothérapeutique avec le mot d'ordre que celui-ci ne vise pas à traiter le trouble de l'identité sexuée [13]. Cependant, avant de présenter et analyser plus en détails les recommandations de la WPATH concernant la psychothérapie des patients transsexuels en transition, il est important de souligner la particularité de l'éventuelle demande de psychothérapie de ceux-ci. En effet, pour les patients, la solution recherchée se situe en premier lieu et avant tout sur les scènes corporelle et sociale, en-dehors de la sphère psychique, et donc en-dehors des capacités d'intervention d'une psychothérapie qui se voit pour sa part attribuer un rôle secondaire [25]. Ainsi, la majorité des patients ne souhaitent pas y recourir [26].

\section{La psychothérapie dans les standards de soins de la World Professional Association for Transgender Health}

Concernant le protocole de THC, à l'heure actuelle, les standards de soins de la WPATH sont considérés comme la référence principale, notamment par l'HAS qui s'est en grande partie inspiré de leur sixième version pour son rapport publié en 2009 [17]. A propos de la psychothérapie, il est reconnu et admis dans ces recommandations et dans ce rapport, qu'un accompagnement psychothérapeutique peut être d'une grande utilité dans le parcours de THC et qu'il peut apporter de réels bénéfices aux patients. Cependant, pour sa part, le rapport de la HAS ne précise pas les modalités et objectifs de cet éventuel accompagnement. De plus, il est important de souligner que comme le rapport publié par l'IGAS [18] qui l'a suivi, le rapport de la HAS [17] se base sur la sixième version des standards de soins de la WPATH, passant à côté du travail de révision vers la septième version de ces standards de soins qui allaient inaugurer le changement majeur qu'est le passage du paradigme du syndrome transsexuel à celui de la santé transgenre, changement qui modifie profondément la prise en charge de ces patients puisqu'il s'agit non plus d'un alignement sur des standards visant le passage d'un sexe à l'autre, mais d'une adaptation des soins de transformation à la demande de chaque patient [8].

\subsection{La psychothérapie dans les premières versions des Standards de soins}

Pour en revenir à la psychothérapie, dès leur première édition en 1979, les standards de soins de la WPATH ont accordé de l'intérêt à une psychothérapie s'ajoutant à la THC et lui ont donc consacré une partie décrivant et précisant ses modalités et objectifs. Cependant, au cours des différentes versions de ces standards, la psychothérapie n'a pas toujours eu le même statut et le même objectif [13]. En effet, de 1979 à 1997, dans les quatre premières versions des standards de soins, la psychothérapie était une étape obligatoire comprenant l'évaluation et la prescription ou non d'une THC par le psychothérapeute. Ces deux éléments étaient d'ailleurs les seuls objectifs donnés à cette psychothérapie. La psychothérapie était donc totalement confondue avec ce qui correspond actuellement à l'étape du diagnostic, de l'évaluation et du suivi, qui en France, reviennent avant tout aux psychiatres parfois assistés de psychologues [15]. Dans cette configuration, la psychothérapie était une condition incontournable imposée aux patients pour qu'ils puissent avoir accès à la THC. Ce n'est qu'en 1998, dans la cinquième version des standards de soins, que la psychothérapie a été clairement différenciée de l'étape du diagnostic, et qu'elle n'a plus été imposée aux patients, comme préalable à la THC. Ainsi, concernant la psychothérapie, il est précisé que le choix et la décision de s'engager dans cette étape reviennent entièrement et uniquement au patient, et que l'objectif de celle-ci n'est pas de traiter le trouble de l'identité sexuée, mais «d'aider [les patients] à 
Marchand J-B. et al. (2017). Transsexualisme/transgenderism : psychothérapie et transformation hormonale et chirurgicale. Annales Médico-Psychologiques, 175 (3), 210-216.

atteindre un confort à long terme vis-à-vis de leur identité de genre, avec des chances réalistes de succès dans leurs relations, éducation et travail. » [21, p. 2]. Cependant, il est ici important de noter que la cinquième version de ces standards ne précise pas explicitement si le psychothérapeute est un professionnel distinct des professionnels participants au diagnostic et à l'évaluation, et ses éventuels relations et son rôle au sein de l'équipe pluridisciplinaire et dans les réunions collégiales. Concernant cette question, actuellement, en France, il est fait état d'accompagnements psychologiques proposés par les équipes pluridisciplinaires et faisant donc partie de la prise en charge par ces équipes, et "parfois [d'orientation vers] un travail psychothérapique par un professionnel extérieur à l'équipe »"

Pour aux standards de soins de la WPATH, en 2001, la sixième version ne comporte pas de changements majeurs concernant la psychothérapie. Cependant, il y est précisé que le psychothérapeute devrait travailler sur l'ensemble de la complexité de la personne et que "[1]es buts de la psychothérapie sont d'aider la personne à vivre plus confortablement dans une identité de genre et de traiter efficacement les problèmes ne concernant pas le genre. » [24]. Or, cet objectif laisse entrevoir que certains patients ne souhaitent pas complètement éradiquer l'intégralité de leurs caractéristiques sexuelles primaires et secondaires. En cela, tous les patients ne correspondraient pas aux patients transsexuels désirant changer sexe tels que décrits par H. Benjamin en 1966 [2], ceci laissant entrevoir implicitement l'introduction du paradigme transgenre. D'un point de vue méthodologique, la section «Psychothérapie » de cette version expose l'importance d'une collaboration étroite entre le psychothérapeute et le patient, dans une perspective qui s'apparente plus au counseling et au coaching qu'à la psychothérapie. Il y s'agit d'aider le patient à trouver un plus grand confort personnel dans la vie quotidienne, notamment à un niveau matériel, par la prescription aux patients de multiples options et procédés pouvant soulager la dysphorie de genre [16], tels que des conseils esthétiques et cosmétiques de travestissements temporaires ou permanents, ainsi que des activités en public ou dans le privé, rappelant, étayant ou affirmant le sexe revendiqué.

\subsection{La septième version des standards de soins : du paradigme du syndrome transsexuel à celui de la santé transgenre et son spectre}

L'édition de la septième version des standards de la WPATH inaugure et concrétise un tournant majeur dans la prise en charge des personnes transsexuelles et transgenres. En effet, deux éléments ont précédé et guidé cette révision : le changement de paradigme et de ligne politique de l'association. En effet, conformément à son changement de nom, la nouvellement nommée WPATH entend promouvoir la santé (telle que définie par l'OMS) des personnes transgenres, abandonnant pour cela le paradigme du transsexualisme qu'elle a reconsidéré comme pathologisant. En comparaison à cet «ancien » paradigme qui était basé sur une conception de l'identité sexuée comme binaire -homme ou femme- avec des personnes qui éprouvaient une souffrance psychique les amenant à demander à changer de sexe, ce nouveau modèle de la santé transgenre abandonne cette dichotomie pour la remplacer par le spectre des variations des multiples identités transgenres considérées comme normales et nécessitant une individualisation et une adaptation des prises en charge de chaque patient, au cas par cas (même si la clinique transgenre reste encore aujourd'hui à préciser [22]).

\footnotetext{
${ }^{4}$ http://www.transsexualisme.info/v2/data/informations/psychiatrie.php
} 
Marchand J-B. et al. (2017). Transsexualisme/transgenderism : psychothérapie et transformation hormonale et chirurgicale. Annales Médico-Psychologiques, 175 (3), 210-216. http://dx.doi.org/10.1016/j.amp.2016.05.016

De même, le second élément ayant joué un rôle important dans l'édition de la nouvelle version des standards de soins est formé de l'ensemble des travaux récents portant sur la psychothérapie des patients transgenres basée sur des modèles explicatifs du développement de l'identité transgenre, qui exclurait une conception pathologique de ce phénomène et expliquerait celui-ci en tant que variation normale. A ce sujet, l'ancienne présidente de la WPATH, Fraser [13] cite comme approches retenues pour établir les fondements théoriques de la psychothérapie des personnes transgenres: l'approche socio-développementale de l'identité (qui comprend le modèle en stades développementaux de Devor et Bockting \& Coleman [3, 12]), l'approche narrative de Lev [20] puis son approche psychodynamique [13]. Succinctement, inspirée directement des modèles socio-développementaux portant sur la formation de l'identité homosexuelle qui mettent l'accent sur l'importance du développement social et sur le rôle des relations interpersonnelles dans le développement de l'identité, l'approche socio-développementale propose des modèles explicatifs en une succession de stades de développement allant de l'émergence de l'identité transgenre jusqu'au coming out et l'affirmation de son identité personnelle [3, 12]. Dans la continuité de celle-ci, l'approche narrative de Lev [20] se fonde sur une approche humaniste qui vise à soutenir l'émergence de l'identité transgenre. Selon les termes de Lev, il s'agit alors pour le psychothérapeute « d'assister à la naissance » du soi du patient transgenre [20]. Le psychothérapeute doit -telle « une sage-femme »- aider et soutenir le patient par des encouragements qui l'aideront à trouver le sens et la raison de ce qu'il a vécu, et qui lui permettront de déterminer la direction et les objectifs de son avenir dans une organisation qui a un sens pour lui [20]. Enfin, de manière cohérente avec ces deux approches, Fraser propose une approche psychodynamique qu'elle revendique non-pathologisante et qui postule que l'identité transgenre serait une partie du vrai Self [13]. Inspirée par Devor, Fraser postule que les problèmes et distorsions dont les patients transgenres seraient victimes, découleraient de défauts de witnessing ${ }^{5}$ et mirroring ${ }^{6}$ $[12,13]$, et/ou de la stigmatisation qui les forceraient à maintenir caché leur genre authentique ce qui entraineraient des difficultés d'ordre psychologique. Selon Fraser, l'impossibilité du patient d'exprimer son genre authentique, entrainerait un défaut de développement de son Moi (pourtant en bonne santé, selon Fraser), défaut de développement du Moi que Fraser relie peut-être de manière un peu hâtive, aux théories psychanalytiques sur rôle de miroir de la mère de Winnicott, sur le transfert en miroir de Kohut ou encore sur le stade du miroir de Lacan [13].

Quoi qu'il en soit, dans ces trois modèles d'approches psychothérapeutiques retenus par la WPATH, il est postulé que les troubles psychologiques que pourraient rencontrer les personnes transgenres ne seraient pas dus à l'identité transgenre elle-même, mais à des problèmes rencontrés dans l'environnement, qui peuvent aller jusqu'à générer un véritable un traumatisme psychique, et que la psychothérapie doit alors traiter par une alliance/empathie inconditionnelle dans la relation thérapeutique.

\subsection{La psychothérapie dans la septième version des standards de soins}

Dans la septième version des standards de soins, la psychothérapie est intégrée à la partie portant sur les "Tâches des professionnels de santé mentale travaillant auprès d'adultes

\footnotetext{
${ }^{5}$ Témoigner au sens de la reconnaissance et de l'assistance.

${ }^{6}$ Traduction : mimétisme comportemental ou comportement de mise en miroir, mécanisme d'apprentissage par synchronisation à un modèle à imiter.
} 
Marchand J-B. et al. (2017). Transsexualisme/transgenderism : psychothérapie et transformation hormonale et chirurgicale. Annales Médico-Psychologiques, 175 (3), 210-216.

présentant une dysphorie de genre ». Il est en premier lieu précisé qu'elle n'est pas un prérequis nécessaire pour avoir accès à l'hormonothérapie et à la chirurgie, même si elle est fortement recommandée. Quelle que soit la forme que prend cette psychothérapie (individuelle, groupale, de couple, familiale...), l'objectif général donné à celle-ci reste : "de trouver des moyens d'optimiser le bien-être psychologique de la personne, sa qualité de vie et son sentiment de réalisation personnelle. La psychothérapie ne vise pas à modifier l'identité de genre de la personne, elle peut l'aider plutôt à explorer les problèmes de genre et à trouver des moyens d'atténuer la dysphorie de genre si elle est présente [...] En règle générale, le but ultime du traitement est d'aider les individus transsexuels, transgenres et de genre nonconforme à atteindre un confort à long terme vis-à-vis de leur identité de genre, avec des chances réalistes de succès dans leurs relations, éducation et travail. » $[8]$

Dans ces standards, changer de genre est plutôt assimilé à un processus psychosocial, et la psychothérapie est décrite comme une aide à celui-ci comprenant le conseil et l'accompagnement des patients transgenres. Il s'agit alors par des échanges interactifs d'aider ces derniers : (1) à clarifier et à explorer leur identité de genre ainsi que leurs rôles de genre, (2) à atténuer l'impact de la stigmatisation et du stress lié à celle-ci sur leur santé mentale et leur développement personnel, (3) et à faciliter le processus de coming-out qui pour certains patients, peut comporter des changements dans l'expression de leurs rôles de genre ainsi qu'un recours à des interventions médicales féminisantes ou masculinisantes.

De manière générale, il s'agit ici par la psychothérapie d'essayer d'apporter un soutien, d'encourager les compétences interpersonnelles et la résilience des personnes transgenres ainsi que de leurs proches, et de soulager les éventuels problèmes psychologiques coexistants. De même, pour les patients qui ont l'intention de changer d'identité de genre de manière définitive, la psychothérapie vise à les aider à planifier un traitement individualisé avec ses buts, ses étapes et sa chronologie, qui prenne en considération ou les aide à prendre conscience que changer d'identité de genre peut avoir d'importantes conséquences aux niveaux social et personnel notamment aux niveaux familial, professionnel, économique et légal. Dans cette perspective, le psychothérapeute peut aider les patients à explorer et à anticiper les conséquences de ces changements d'identité, offrir un espace aux patients pour qu'ils puissent commencer à s'exprimer en harmonie avec leur identité de genre, et pour aider certains patients à surmonter leurs craintes liées à ces changements, entre autre avec la programmation de risques calculés en-dehors de la thérapie leur permettant de gagner en expérience et en confiance dans l'expression de leur identité, ainsi qu'avec un accompagnement au coming-out dans la famille ou les divers domaines de leur vie. Pour d'autres personnes transgenres qui se présentent après avoir déjà entamé une expérience en vie réelle dans leur identité de genre revendiquée (aussi minime et temporaire soit-elle), la psychothérapie peut les aider à identifier et travailler sur les problèmes potentiels et aussi à favoriser un ajustement optimal de leur changement d'expression d'identité de genre.

Enfin, les standards de soins insistent sur le fait que le travail psychothérapeutique peut prendre la forme d'un suivi tout au long de la vie, et qu'il peut aussi être nécessaire de prendre en considération d'autres domaines que le changement d'identité de genre, comme sa famille (notamment avec une thérapie -ou un soutien- familiale), ou encore -si le patient le demande- une exploration de son orientation sexuelle et de ses fantasmes érotiques.

Pour finir, il est important de noter qu'au sujet du praticien, la septième version des standards de soins de la WPATH ne parle pas spécifiquement de «psychothérapeutes » mais de manière 
Marchand J-B. et al. (2017). Transsexualisme/transgenderism : psychothérapie et transformation hormonale et chirurgicale. Annales Médico-Psychologiques, 175 (3), 210-216. http://dx.doi.org/10.1016/j.amp.2016.05.016

beaucoup plus large, de «professionnels de santé mentale»dont la psychothérapie est une des tâches qui leur incombent en plus de celles d'évaluation et d'orientation. De même, ces standards insistent sur la nécessité d'entretenir autant que possible une collaboration avec les autres professionnels de santé de l'équipe pluridisciplinaire et si possible d'assister et de participer régulièrement à des synthèses et séances de coordination avec les autres professionnels de l'équipe pluridisciplinaire.

\section{Remarques sur la prise en charge psychothérapeutique des personnes transgenres et sa mise en relation avec la Transformation Hormonale et Chirurgicale}

A l'issue de cette description de la psychothérapie selon les standards de soins de la WPATH, quelques remarques et interrogations se rapportant à celle-ci semblent mériter d'être faites notamment aux niveaux clinique, pratique et théorique. En effet, au-delà de la présentation et de la description de la psychothérapie, la mise en place d'une psychothérapie dans le protocole de THC reste un élément du parcours de soins porteur d'ambiguités et pouvant semer une certaine confusion. Avant d'entrer dans le détail de ces soucis, on peut souligner brièvement que les définitions du cadre et des objectifs de cette psychothérapie restent problématiques.

\subsection{La demande du patient}

En premier lieu, il convient de préciser le contexte particulier dans lequel s'entremêlent la demande des patients d'accéder à une THC, et la proposition d'une psychothérapie au sein de ce parcours de soins. En effet, les patients ont une position et une approche particulière de leur souffrance qu'ils n'estiment pas en lien avec une quelconque dimension psychique. Ils amènent leur autodiagnostic et demandent avant tout un accès à la THC sollicitant donc en premier lieu et s'ils le pouvaient exclusivement, les endocrinologues et chirurgiens. Ainsi, comme le souligne A. Michel [26], ils ne sont que très rarement intéressés par un travail personnel, et peuvent parfois se méfier des professionnels de santé mentale et de l'éventualité que ces derniers peuvent se faire ou avoir un avis négatif concernant leur demande.

Dans cette configuration, il est important comme le recommandent la WPATH et l'HAS que la psychothérapie ne soit pas imposée aux patients, et que l'objectif de la psychothérapie reste d'apporter un soutien au patient tout au long et si nécessaire au-delà de la THC. Elle ne doit pas être une condition nécessaire pour l'accès à la THC, et la décision d'entreprendre ce travail thérapeutique doit uniquement revenir au patient. Cependant, cette proposition de psychothérapie reste importante. La problématique identitaire, les changements physiques, sociaux et relationnels induits par la THC peuvent s'accompagner ou engendrer une grande vulnérabilité et une importante détresse psychologique qui doivent être prises en compte et soulagées par un soutien psychothérapeutique adapté. Or, le fait que la proposition d'un «accompagnement» soit faite par l'équipe pluridisciplinaire ne met pas le patient dans la position de pouvoir librement choisir de s'engager ou non dans ce soin, et ce d'autant plus si ce soin se fait avec un professionnel membre de l'équipe pluridisciplinaire. Dans ces conditions, le patient peut se sentir obliger de s'engager dans une psychothérapie pensant ainsi convenir à l'équipe pluridisciplinaire et obtenir la THC.

\subsection{Détermination du professionnel psychothérapeute}


Marchand J-B. et al. (2017). Transsexualisme/transgenderism : psychothérapie et transformation hormonale et chirurgicale. Annales Médico-Psychologiques, 175 (3), 210-216.

A ce sujet, parler d'un «accompagnement» psychothérapeutique plutôt que d'une psychothérapie, n'est pas sans ambigüité, car se pose la question du professionnel à qui confier le rôle de psychothérapeute et celle de l'intégration ou non de celui-ci à l'équipe pluridisciplinaire et aux réunions collégiales. Qu'il s'agisse d'un psychiatre ou d'un psychologue, les professionnels de santé mentale qui sont déjà engagés dans l'évaluation et le diagnostic, ne devraient pas prendre ce rôle. En effet, le premier rôle qu'ils ont joué risque de gêner l'établissement d'un cadre neutre et de confiance propice à un travail psychothérapeutique. De plus, le mélange des rôles peut être à l'origine de confusions et être déstabilisant pour le professionnel comme pour le patient. Du point de vue du patient, celui-ci peut ne pas parvenir à prendre de la distance quant au rôle premier d'évaluateur du professionnel et donc ne pas se sentir dans les conditions lui permettant de parler sans crainte que ses paroles aient des conséquences sur le déroulement de la THC. De même, du point de vue du professionnel, ce dernier se retrouve à avoir un double statut d'évaluateurpsychothérapeute dont les objectifs restent différents. Par ailleurs, ce double statut peut avoir une influence sur le choix supposé libre du patient d'entreprendre ou non une psychothérapie. Toutefois, d'un point de vue matériel, il faut reconnaitre que les effectifs et les moyens dont disposent les équipes pratiquant la THC, restent limités et que dès lors la mise en place d'une telle condition ne peut le plus souvent pas être respectée même si nécessaire. En effet, les effectifs des équipes françaises sont souvent trop restreints pour qu'un psychologue ou un psychiatre ne puisse consacrer tout ou partie de son temps à la psychothérapie des patients, sans jamais avoir à tenir un autre rôle dans l'évaluation et le suivi du parcours de soins de ces mêmes patients. De même, il n'est pas simple d'orienter les patients vers d'autres lieux de consultations car peu de professionnels sont formés à la prise en charge de ces patients.

\subsection{La question de l'intégration du psychothérapeute au travail de l'équipe pluridisciplinaire}

Par ailleurs, cette dernière remarque appelle un autre point qu'il est important d'aborder concernant la psychothérapie, à savoir l'intégration ou non du psychothérapeute au sein de l'équipe pluridisciplinaire et surtout sa présence aux réunions portant sur l'avancement de la THC. En effet, de cette interrogation qui ne trouve pas de réponse consensuelle, il découle plusieurs questions primordiales pouvant avoir des conséquences sur la qualité du travail psychothérapeutique ainsi que sur le déroulement du parcours de soins. A partir de cette question, il est légitime de se demander si le psychothérapeute ne glisse pas dans le même double positionnement problématique que les professionnels ayant participé aux étapes de diagnostic et d'évaluation. La psychothérapie peut être influencée par l'avancement et les objectifs de la THC, ou alors se confondre et à l'inverse agir sur le déroulement de la THC, notamment si le psychothérapeute doit émettre un avis sur l'état du patient et sur le déroulement de la psychothérapie avant le passage à une autre étape de la THC. Concernant ce dilemme, les solutions proposées se résument à deux positions. La première position majoritaire est que le psychothérapeute qu'il fasse partie de l'équipe pluridsciplinaire ou non, doit travailler en étroite collaboration avec celle-ci [8, 17]. Puis, une seconde position minoritaire défendue par Chiland [7], selon laquelle, dans le but de préserver le cadre de la psychothérapie, celle-ci de même que le psychothérapeute ne doivent au contraire ni faire partie de l'équipe pluridisciplinaire, ni participer au déroulement de la THC. Bien que différentes, ces deux positions cherchent toutes deux à mettre en place les meilleures conditions d'obtention de prise en charge. La différence est que la première privilégie le bon 
Marchand J-B. et al. (2017). Transsexualisme/transgenderism : psychothérapie et transformation hormonale et chirurgicale. Annales Médico-Psychologiques, 175 (3), 210-216. http://dx.doi.org/10.1016/j.amp.2016.05.016

déroulement et l'aboutissement de la THC, alors que la seconde, préserve un cadre neutre plus propice au travail psychothérapeutique.

\subsection{Méthode et objectifs de la prise en charge psychothérapeutique}

De même, il convient aussi de s'interroger sur la méthode psychothérapeutique ainsi que ses objectifs. En effet, comme le souligne la HAS [17], il est difficile d'établir un schéma psychothérapeutique standard, et on peut constater que dans la littérature portant sur le transsexualisme peu de publications concernent spécifiquement la prise en charge psychothérapeutique. Toutefois, il convient de préciser quelques points supplémentaires concernant cette prise en charge. En effet, en plus du fait que peu de ces patients s'orientent vers un travail psychothérapeutique, il faut souligner le postulat atypique proclamé par la WPATH selon lequel, dans le cas d'une prise en charge psychothérapeutique de ces patients, la psychothérapie ne doit pas porter sur l'identité sexuée [9]. De manière assez particulière en comparaison avec d'autres prises en charge psychothérapeutiques, celle-ci n'a pas pour objectif de traiter le trouble de l'identité sexuée ce qui correspondrait à essayer d'aider le patient à se réconcilier avec son sexe de naissance. Au contraire, certains conseils de la WPATH concernant la psychothérapie, proches du coaching et/ou du counseling, vont dans le sens d'un encouragement de l'expression d'une nouvelle identité de genre. Or, il est ici pertinent de souligner le caractère paradoxal de cette recommandation. L'identité sexuée est l'objet de la consultation du patient. Sans pour autant s'opposer à ce discours, il est important de ne pas nier et s'interdire d'aborder ce sujet central dans la vie et le fonctionnement psychique du patient, ainsi que son éventuelle dimension conflictuelle. Plutôt que cette interdiction, il parait plus approprié d'adopter une position de neutralité bienveillante, même si celle-ci peut être mise à mal par la demande des patients et la THC (qui selon Oppenheimer, peut agir dans la psychothérapie tel un acting out permanent [28]). Comme le faisait remarquer J. McDougall à une autre époque, concernant la psychanalyse des analysants homosexuels, le psychanalyste doit se défaire de toute attente et de tout apriori concernant l'orientation sexuelle de l'analysant, et la psychanalyse ne doit ni avoir pour objectif d'aller à l'encontre du choix d'objet du patient, ni encourager ce choix [23].

Pour conclure sur ces aspects concrets de la psychothérapie, il convient de garder à l'esprit que les remarques concernant la psychothérapie des standards de soins de la WPATH sont pertinents. Ils révèlent des éléments cliniques intéressants et témoignent d'une certaine expérience de prises en charge des patients transsexuels et transgenres, mais il est aussi nécessaire de ne pas en faire un guide prédéfini et surtout trop rigide. Pour cela, le psychothérapeute doit donc connaitre ces recommandations, mais aussi les critiques et les limites de celles-ci, et continuer de s'interroger sur sa pratique dans le contexte du protocole de THC, et face aux demandes de ces patients.

\section{Pour conclure : nécessité de poursuivre la recherche de modèles explicatifs du transsexualisme et du transgenderism}

Enfin, concernant les modèles psychothérapeutiques présentés ci-dessus, nous remarquons qu'il s'agit de modèles a-conflictuels qui orientent la psychothérapie vers un étayage thérapeutique. Or, les problématiques identitaires transsexuelles et transgenres ni ne se limitent, ni ne se restreignent à un processus psychosocial, et ne sont pas dépourvues de conflictualité. Ainsi, pour améliorer la prise en charge psychothérapeutique proposée à ces patients, il nous faut donc continuer de réfléchir au-delà des carcans de la psychopathologie 
Marchand J-B. et al. (2017). Transsexualisme/transgenderism : psychothérapie et transformation hormonale et chirurgicale. Annales Médico-Psychologiques, 175 (3), 210-216. http://dx.doi.org/10.1016/j.amp.2016.05.016

structurale et de l'approche déconstructiviste, et ce par une approche et des éléments théoriques eux-mêmes transversaux. En effet, les phénomènes transsexuel et transgenre invitent à penser la subjectivité en situant cette dernière au carrefour des mouvements du corporel, du socioculturel et de l'intrapsychique. Parmi ces éléments théoriques transversaux, nous pensons en premier lieu aux travaux de Laplanche sur le «Sexual » [19]; et en second lieu, à la troisième topique de Dejours [11] -cette dernière nous permettant de rendre compte d'une partie de l'inconscient différente de l'inconscient refoulé. En effet, se rapprochant d'un subconscient, cette autre partie nommée inconscient « amential » s'exprimerait différemment de celle relevant du refoulé, dans un rapport à la réalité plus direct. D'autre part, nous pouvons mentionner la clinique de l'expérience subjective du corps de l'hypocondriaque par Brusset qui nous permet de réfléchir au niveau de la prise en charge de ces patients sujets à des projections intracorporelles [4, 5] qui cherchent une réponse en-dehors de la psychothérapie, situation dans laquelle Brusset recommande au psychothérapeute de ne pas s'immiscer, ni se confondre avec la position médicale, et de se positionner en tiers neutre externe et distinct du corps médical. Enfin, entrant dans le cadre de la clinique de l'extrême [30], les phénomènes transsexuel et transgenre engagent un discours et un parcours extraordinaires avec des difficultés et des transformations plus ou moins importantes appelant à un grand nombre de mouvements et remaniements internes tant chez le patient que du point de vue du psychothérapeute. Or, face à l'extrême, il incombe aux deux protagonistes de travailler sur l'image qui habite et accompagne le patient, image qui suscite chez lui la peur et l'angoisse. Dans un deuxième temps, suite à ce travail de déconstruction, il s'agirait ensuite pour le psychothérapeute de convertir cette image en une scène partageable qui ne concerne dès lors non plus seulement le patient, mais aussi le praticien qui devrait alors devenir tel un matériau modelé et modelable [30]. Enfin, dans une telle position et par ses interprétations autour d'un signifiant, le psychothérapeute permettrait alors au patient confronté à l'extrême de retrouver une partie de son moi idéal, avec un moi expansé tout-puissant incluant l'autre (à savoir le psychothérapeute). Les interventions viendraient alors ressourcer le narcissisme du patient et rendre possible l'engagement d'un processus de reconstruction.

\section{Bibliographie}

[1] American Psychiatric Association. Diagnostic and Statistical Manual of mental disorders (5th ed.- DSM 5). Arlington, VA : American Psychiatric Publishing ; 2013 ; 451-59.

[2] Benjamin H. The transsexual phenomenon. New York : Julian Press ; 1966.

[3] Bockting W, et Coleman E. Developmental stages of the transgender coming-out process. In Ettner R, Monstrey S, et Eyler A. Principles of transgender medicine and surgery. New York : Haworth Press ; 2007 : 185-208.

[4] Brusset B. L'hypocondrie : thématique ou organisation spécifique ?. Revue française de psychosomatique $2002 ; 2, \mathrm{n}^{\mathrm{o}} 22: 45-64$. [le 26 mars 2015, www.cairn.info/revue-francaisede-psychosomatique-2002-2-page-45.htm ; doi : 10.3917/rfps.022.0045]

[5] Brusset B. Au-delà de la névrose - vers une troisième tropique. Paris : Dunod ; 2013.

[6] Castel P-H. La métamorphose impensable, essai sur le transsexualisme et l'identité personnelle. Paris : Gallimard ; 2003.

[7] Chiland C. Changer de sexe. Paris : Odile Jacob ; 1997, 2011.

[8] Coleman E, et al. Standards of Care for the Health of Transsexual, Transgender, and Gender-Nonconforming People, Version 7, International Journal of Transgenderism 2011 ; 13, 4 ; 165-232. doi:10.1080/15532739. 2011.700873. [le 26 mars 2015, (dont traduction française : Standards de Soins pour la santé des personnes transsexuelles, transgenres et de 
Marchand J-B. et al. (2017). Transsexualisme/transgenderism : psychothérapie et transformation hormonale et chirurgicale. Annales Médico-Psychologiques, 175 (3), 210-216.

genre non-conforme), http://www.wpath.org/site_page.cfm?pk_association_webpage_menu= 1351\&pk_association_webpage $=4655]$

[9] Czermak M. Précisions sur la clinique du transsexualisme. In Les Passions de l'objet. Paris : Clims ; $1986 ; 109-28$.

[10] Czermak M, Frignet H, et al. Sur l'identité sexuelle : à propos du transsexualisme. Paris : Association Freudienne Internationale ; 1996.

[11] Dejours C. Le corps d'abord. Paris : Payot ; 2001.

[12] Devor A. Witnessing and mirroring : A fourteen stage model of transsexual identity formation, In Leli U, Drescher J, Transgender subjectivities: A clinician's guide. Binghamton, NY : Haworth Press ; 2004.

[13] Fraser L. Psychotherapy in the World Professional Association for Transgender Health's Standard of Care: Background and Recommandations, International Journal of Transgenderism $2009 ; 11: 110-26$.

[14] Frignet H. Le transsexualisme. Paris : Desclée de Brouwer ; 2000.

[15] Gorin-Lazard A, Bonierbale M, Magaud-Vouland N, Michel A, Morange I, Perchenet A-S et al. Trouble de l'identité de genre : quel est le rôle du psychiatre ?. Sexologies 2008 ; $17: 225-37$.

[16] Harry Benjamin International Gender Dysphoria Association. Standards of care for gender identity disorders, sixth version, Minneapolis, 2001.

[17] Haute Autorité de Santé. Situation actuelle et perspectives d'évolution de la prise en charge médicale du transsexualisme en France. 2009 avril. version pour consultation publique-avril 2009. [le 26 mars 2015, http://www.has-sante.fr/portail/upload/docs/ application/pdf/2009-12/rapport_transsexualisme.pdf]

[18] Inspection Générale des Affaires Sociales. Evaluation des conditions de prise en charge médicale et sociale des personnes trans et du transsexualisme, Rapport $\mathrm{N}^{\circ} \mathrm{RM} 20112011-197 \mathrm{P}$, 2011. [le 26 mars 2015, http://www.ladocumentationfrancaise.fr/var/storage/rapportspublics/124000209/0000.pdf]

[19] Laplanche J. Sexual, la sexualité élargie au sens freudien. Paris : PUF ; 2007.

[20] Lev A. Transgender emergence: Therapeutic guidelines for working with gender-variant people and their families. New York : Haworth Clinical Practice Press ; 2004.

[21] Levine S, Brown G, Coleman E, Cohen-Kettenis P, Hage J, Van Maasdam, J, et al. The standards of care for gender identity disorders. International Journal of Transgenderism $1998 ; 5,1,2,2$.

[22] Marchand J-B, Pelladeau E, Pommier F. Du transsexualisme à la dysphorie de genre : regroupement ou amalgame. Evolution Psychiatrique 2015; 80, 2: 331-48. [doi : 10.1016/j.evopsy.2015.01.006].

[23] McDougall J. Plaidoyer pour une certaine anormalité. Mayenne : Gallimard ; 1978.

[24] Meyer III W, Bockting W, Cohen-Kettenis P, Coleman E, Di Ceglie D, Devor A, et al. The standards of care for gender identity disorders - Sixth version. International Journal of Transgenderism $2001 ; 5,1$.

[25] Michel A. Le transsexuel : quel devenir ?, Annales médico-psychologiques $2001 ; 159$ : $347-58$.

[26] Michel A. Les troubles de l'identité sexuée. Paris ; Armand Colin ; 2006.

[27] Morel G. Ambiguittés sexuelles. Sexuation et psychose. Paris : Anthropos ; 2000.

[28] Oppenheimer A. Le désir de changer de sexe: un défi pour la psychanalyse?. Psychanalyse à l'université $1992 ; 17,66$ : 117-34. 
Marchand J-B. et al. (2017). Transsexualisme/transgenderism : psychothérapie et transformation hormonale et chirurgicale. Annales Médico-Psychologiques, 175 (3), 210-216. http://dx.doi.org/10.1016/j.amp.2016.05.016

[29] Organisation Mondiale de la Santé. Troubles de l'identité sexuelle. In Classification statistique internationale des maladies et des problèmes de santé connexes $10 \mathrm{e}$ version (CIM 10), Chapitre V. Genève ; 1993 : p. 123.

[30] Pommier F. L'extrême. In Marty F, et al. Les grandes problématiques en psychologie clinique. Paris : Dunod ; 2009 : 145-60. 